\title{
Global Existence for Systems of Nonlinear Wave Equations in Two Space Dimensions
}

By

\section{Soichiro KATAYAMA *}

\section{§1. Introduction}

We consider the Cauchy problem for systems of fully nonlinear wave equations of the type

$$
\begin{gathered}
\square u_{i}=F_{i}\left(u, u^{\prime}, u^{\prime \prime}\right) \quad \text { in } \overline{\boldsymbol{R}}_{+} \times \boldsymbol{R}^{n}, \quad i=1, \cdots, N, \\
u_{i}(0, x)=\varepsilon f_{i}(x), \partial_{t} u_{i}(0, x)=\varepsilon g_{i}(x), \quad x \in \boldsymbol{R}^{n}, i=1, \cdots, N,
\end{gathered}
$$

where $\partial_{0}=\partial_{t}=\partial / \partial t, \partial_{j}=\partial / \partial x_{j}, j=1, \cdots, N, \square=\partial_{t}^{2}-\sum_{i=1}^{n} \partial_{i}^{2}$ is the D'Alembertian, $F=\left(F_{j}\right), \quad u=\left(u_{j}\right), \quad u^{\prime}=\left(u_{j, a}\right)=\left(\partial_{a} u_{j}\right), u^{\prime \prime}=\left(u_{j, a b}\right)=$ $\left(\partial_{a} \partial_{b} u_{j}\right)$ with $j=1, \cdots, N$ and $a, b=0,1, \cdots, n$. Let $f_{i}, g_{i} \in C_{0}^{\infty}\left(\boldsymbol{R}^{n}\right)$ for $i=$ $1, \cdots, N$ and $\varepsilon>0$ be a small parameter.

We assume that $F$ is a smooth function in its arguments satisfying $F=$ $O\left(|u|^{p}+\left|u^{\prime}\right|^{p}+\left|u^{\prime \prime}\right|^{p}\right)$ near $\left(u, u^{\prime}, u^{\prime \prime}\right)=0$ with some positive integer $p$ and

(A1) $\frac{\partial F_{i}}{\partial u_{j, a b}}=0$ for all $j \neq i$ and $a, b=0, \cdots, n$.

Let $T_{\varepsilon}$ be the life-span of the classical solution to (1.1)-(1.2). When $F$ does not depend on $u$ explicitly, namely $F=F\left(u^{\prime}, u^{\prime \prime}\right)$, the following results are known: When $n=3, T_{\varepsilon} \geq \exp \left\{c \varepsilon^{-1}\right\}$ for $p=2, T_{\varepsilon}=+\infty$ for $p \geq 3$, and when $n=2, T_{\varepsilon} \geq c \varepsilon^{-2}$ for $p=2, T_{\varepsilon} \geq \exp \left\{c \varepsilon^{-2}\right\}$ for $p=3$ and $T_{\varepsilon}=+\infty$ for $p \geq 4$, if $\varepsilon$ is sufficiently small. (See Klainerman [7], Kovalyov [9], Li-Yu [12].)

In general cases it is known that when $n=3, T_{\varepsilon} \geq c \varepsilon^{-2}$ for $p=2$ and $T_{\varepsilon}=+\infty$ for $p \geq 3$. Klainerman [8] showed that when $n=3$ and $p=2$, if the quadratic part of nonlinear terms satisfies the null condition (which will be

Communicated by T. Kawai, December 25, 1992.

1991 Mathematics Subject Classifications : 35L70.

* Department of Applied Mathematics and Physics, Kyoto University, Kyoto 606, Japan. 
stated later), then $T_{\varepsilon}=+\infty$ for sufficiently small $\varepsilon$. See also Christodoulou [1] and John [5].

When $n=2$, it was shown that $T_{\varepsilon} \geq c \varepsilon^{-6}$ for $p=3$, and $T_{\varepsilon}=+\infty$ for $p \geq 4$. (See $\mathrm{Li}-\mathrm{Yu}-\mathrm{Zhou}[13]$. In fact they are concerning single equations, but these results also hold for systems.)

Recently Godin [2] showed that for single semilinear wave equations in two space dimensions of the type

$$
\square u=F\left(u^{\prime}\right) \quad \text { in } \overline{\boldsymbol{R}}_{+} \times \boldsymbol{R}^{2}, F\left(u^{\prime}\right)=O\left(\left|u^{\prime}\right|^{2}\right) \quad \text { near } u^{\prime}=0 \text {, }
$$

$T_{\varepsilon} \geq \exp \left\{c / \varepsilon^{-2}\right\}$ when the quadratic part of $F$ satisfies the null condition, and $T_{\varepsilon}=+\infty$ when the quadratic and the cubic part of $F$ satisfy the null condition.

His proof is using some transformation associated with the null condition to treat the quadratic term. It works well for single and semilinear equations, but it is not applicable to the system (1.1). So we only treat the case $n=2, p=3$ for the system in this paper, and we prove the global existence under certain assumptions, following the method used in Klainerman [8].

\section{§ 2. Klainerman's Null Condition and the Main Theorem}

In this section we state about Klainerman's null condition (see Klainerman [8] and Christodoulou [1]) and our main theorem.

Definition 2.1. Let $F$ be a smooth function of $u=\left(u_{i}\right), v=\left(v_{i, a}\right)$ and $w$ $=\left(w_{i, a b}\right)$ with $i=1, \cdots, N$ and $a, b=0,1, \cdots, n$. We say that $F$ satisfies the null condition when

$$
F\left(\lambda_{i}, \mu_{i} X_{a}, \nu_{i} X_{a} X_{b}\right)=0
$$

for all $\lambda, \mu, \nu \in \boldsymbol{R}^{N}$ and all $X=\left(X_{0}, X_{1}, \cdots, X_{n}\right) \in \boldsymbol{R}^{n+1}$ such that $X_{0}^{2}-X_{1}^{2}-\cdots$ $-X_{n}^{2}=0$.

We assume that $F$ in (1.1) satisfies the following :

$$
\begin{aligned}
& F=O\left(|u|^{3}+\left|u^{\prime}\right|^{3}+\left|u^{\prime \prime}\right|^{3}\right) \quad \text { near }\left(u, u^{\prime}, u^{\prime \prime}\right)=0, \\
& F(u, 0,0)=O\left(|u|^{5}\right), \\
& F_{i}\left(u, u^{\prime}, u^{\prime \prime}\right)=G_{i}\left(u, u^{\prime}, u^{\prime \prime}\right)+H_{i}\left(u, u^{\prime}, u^{\prime \prime}\right), i=1, \cdots, N,
\end{aligned}
$$


where $G_{i}$, the cubic term of $F_{i}$, satisfies the null condition, and $H_{i}=O\left(|u|^{4}+\left|u^{\prime}\right|^{4}+\left|u^{\prime \prime}\right|^{4}\right)$ near $\left(u, u^{\prime}, u^{\prime \prime}\right)=0$.

Without loss of generalities we can also assume that

(A5) (1. 1) is quasi-linear, i. e., $F_{i}$ is linear in $\left(u_{i, a b}\right)$ for $i=1, \cdots, N$.

Now we can state our main theorem.

Theorem 1. Let $n=2$. Assume $F$ satisfies (A1)-(A4), then there exists a positive constant $\varepsilon_{0}$ (depending on $f, g, F$ ) such that for any $\varepsilon \leq \varepsilon_{0}$, a smooth solution $u(t, x)$ to (1. 1)-(1.2) exists for $0 \leq t<+\infty$.

The proof of this theorem will be given in section 4 .

Remark 1. If we only assume (A1)-(A3), it was proved in $\mathrm{Li}-\mathrm{Yu}-\mathrm{Zhou}$ [13] that $T_{\varepsilon} \geq \exp \left\{c \varepsilon^{-2}\right\}$. But it is not known whether this result is sharp or not.

Remark 2. For simplicity we assumed that the initial conditions depend linearly on the parameter $\varepsilon$, but we can show all the results mentioned above, if we replace (1.2) by the following :

$$
u_{i}(0, x)=f_{i}(x ; \varepsilon), \partial_{t} u_{i}(0, x)=g_{i}(x ; \varepsilon), \quad i=1, \cdots, N
$$

where $f_{i}, g_{i} \in C^{\infty}\left(\boldsymbol{R}^{2} \times \overline{\boldsymbol{R}}_{+}\right), f_{i}(x ; 0)=g_{i}(x ; 0)=0$ and $f_{i}(x ; \varepsilon)=g_{i}(x ; \varepsilon)$ $=0$ for $|x|>R$ with some $R>0$.

Following Klainerman [7], we introduce $\Omega_{0 j}=t \partial_{j}+x_{j} \partial_{t}$ for $j=1,2$, $\Omega_{12}=x_{1} \partial_{2}-x_{2} \partial_{1}, \Omega_{b a}=-\Omega_{a b}$ for $0 \leq a<b \leq 2$, and $\Gamma_{0}=t \partial_{t}+\sum_{j=1}^{2} x_{j} \partial_{j}$. Then it follows that

$$
\left[\Gamma_{0}, \square\right]=-2 \square,\left[\Omega_{a b}, \square\right]=0 \text { for any } a, b=0,1,2 \text {. }
$$

Let $\eta=\left(\eta_{a b}\right)_{a, b=0,1,2}=\operatorname{diag}(-1,1,1)$, then one can easily verify that

$$
\left[\Omega_{a b}, \partial_{c}\right]=\eta_{a c} \partial_{b}-\eta_{b c} \partial_{a},
$$

$$
\left[\Omega_{a b}, \Omega_{c d}\right]=\eta_{a c} \Omega_{b d}-\eta_{b c} \Omega_{a d}+\eta_{a d} \Omega_{b c}-\eta_{b d} \Omega_{a c},
$$

$$
\left[\Omega_{a b}, \Gamma_{0}\right]=0
$$




$$
\left[\Gamma_{0}, \partial_{a}\right]=-\partial_{a}
$$

Let $\Gamma_{1}=\Omega_{01}, \Gamma_{2}=\Omega_{02}, \Gamma_{3}=\Omega_{12}, \Gamma_{4}=\partial_{t}, \Gamma_{5}=\partial_{1}, \Gamma_{6}=\partial_{2}$. We write $\Gamma^{\alpha}$ $=\Gamma_{0}^{\alpha_{0}} \Gamma_{1}^{\alpha_{1}} \cdots \Gamma_{6}^{\alpha_{6}}$ for any multi-index $\alpha=\left(\alpha_{0}, \cdots, \alpha_{6}\right)$. From (2.3)-(2.6) we find that

$$
\Gamma^{\alpha} \Gamma^{\beta} u=\Gamma^{\alpha+\beta} u+\sum_{|\gamma| \leq k+l-1} \mathrm{C}_{r}^{\alpha \beta} \Gamma^{\gamma} u
$$

for $|\alpha|=k,|\beta|=l$, and that

$$
\Gamma^{\alpha} \partial_{a} u=\partial_{a} \Gamma^{\alpha} u+\sum_{b=0}^{2} \sum_{|\beta| \leq k-1} C_{\beta b}^{\alpha a} \partial_{b} \Gamma^{\beta} u(t, x)
$$

for $|\alpha|=k, a=0,1,2$, where $C_{\gamma}^{\alpha \beta}$ and $C_{\beta b}^{\alpha a}$ are appropriate constants.

If $u(t, x)$ satisfies $\square u(t, x)=f(t, x)$, then (2.2) leads to

$$
\square\left(\Gamma^{\alpha} u\right)=\Gamma^{\alpha} f(t, x)+\sum_{|\beta| \leq k-1} C_{\beta}^{\alpha} \Gamma^{\beta} f(t, x)
$$

for $|\alpha|=k$.

For any integer $k$ and for any scalar or vector valued function $u(t, x)$, define

$$
\begin{aligned}
|u(t, x)|_{k} & =\sum_{|\alpha| \leq k}\left|\Gamma^{\alpha} u(t, x)\right|, \\
|\partial u(t, x)|_{k} & =\sum_{|\alpha| \leq k} \sum_{a=0}^{2}\left|\Gamma^{\alpha} \partial_{a} u(t, x)\right|, \\
\|u(t)\|_{p} & =\|u(t, \bullet)\|_{p}=\left(\int_{R^{2}}|u(t, x)|^{p} d x\right)^{1 / p} \text { for } 1 \leq p<+\infty, \\
\|u(t)\|_{\infty} & =\|u(t, \bullet)\|_{\infty}=\sup _{x \in R^{2}}|u(t, x)|, \\
\|u(t)\|_{p, k} & =\|u(t, \cdot)\|_{p, k}=\left(\int_{R^{2}}|u(t, x)|_{k}^{p} d x\right)^{1 / p} \text { for } 1 \leq p<+\infty, \\
\|u(t)\|_{\infty, k} & =\|u(t, \circ)\|_{\infty, k}=\sup _{x \in R^{2}}|u(t, x)|_{k}, \\
\|\partial u(t)\|_{2, k} & =\|\partial u(t, \circ)\|_{2, k}=\left(\int_{R^{2}}|\partial u(t, x)|_{k}^{2} d x\right)^{1 / 2} .
\end{aligned}
$$

In the remainder of this section we state some results concerning the null condition. For any smooth functions $f, g$, define

$$
Q_{a b}(f, g)=\partial_{a} f \partial_{b} g-\partial_{b} f \partial_{a} g \text { for } a, b=0,1,2
$$

and 


$$
Q(f, g)=\partial_{t} f \partial_{t} g-\sum_{j=1}^{2} \partial_{j} f \partial_{j} g .
$$

These functions are closely connected with the null condition. (See Klainerman [8]. )

In fact, suppose $F=\left\{F_{i}\left(u, u^{\prime}, u^{\prime \prime}\right)\right\}_{i=1, \cdots, N}$ satisfies the assumptions (A1)(A5). Then one can write $G_{i}$, which is the cubic part of $F_{l}$, as

$$
\begin{aligned}
G_{i}\left(u, u^{\prime}, u^{\prime \prime}\right)= & \sum_{j, k} P_{i}^{j k}\left(u, u^{\prime}, u^{\prime \prime}\right) Q\left(u_{j}, u_{k}\right) \\
& +\sum_{a, b, j, k} P_{i}^{a b j k}\left(u, u^{\prime}, u^{\prime \prime}\right) Q_{a b}\left(u_{j}, u_{k}\right) \\
& +\sum_{a, j} P_{l, a}^{J}\left(u, u^{\prime}\right) Q\left(u_{j}, \partial_{a} u_{i}\right) \\
& +\sum_{a, b, c, j} P_{i, c}^{a b j}\left(u, u^{\prime}\right) Q_{a b}\left(u_{\jmath}, \partial_{c} u_{l}\right),
\end{aligned}
$$

where $P_{i}^{j k}, P_{l}^{a b j k}, P_{l, a}^{J}$ and $P_{i, c}^{a b j}$ are linear combinations of their arguments and

$$
\frac{\partial P_{i}^{j k}}{\partial u_{l, c d}}=\frac{\partial P_{i}^{a b j k}}{\partial u_{l, c d}}=0 \quad \text { for all } l \neq i
$$

The following lemma due to Klainerman [8] is essential for our proof of the theorem.

Lemma 2. 2. For any integer $k \geq 0$, there exists a constant $C_{k}$ such that

$$
\begin{aligned}
& \text { (i ) } \begin{aligned}
\left|\Gamma^{\alpha} Q_{a b}(f, g)\right| & C_{k}(1+t)^{-1}\left(|f(t, x)|_{[k / 2]+1}|g(t, x)|_{k+1}\right. \\
+ & \left.|g(t, x)|_{[k / 2]+1}|f(t, x)|_{k+1}\right), \quad a, b=0,1,2,
\end{aligned} \\
& \text { (ii) }\left|\Gamma^{\alpha} Q(f, g)\right| \leq C_{k}(1+t)^{-1}\left(|f(t, x)|_{[k / 2]+1}|g(t, x)|_{k+1}\right. \\
& \left.+|g(t, x)|_{[k / 2]+1}|f(t, x)|_{k+1}\right)
\end{aligned}
$$

for any $|\alpha|=k$ and any smooth functions $f, g$.

Proof. For $|\alpha|=k$, we can show that

$$
\begin{aligned}
\Gamma^{\alpha} Q_{a b}(f, g)=Q_{a b}\left(\Gamma^{\alpha} f, g\right)+Q_{a b}\left(f, \Gamma^{\alpha} g\right) \\
\quad+\sum_{c, d=0}^{2} \sum_{|\beta|+|\gamma| \leq k-1} \mathrm{C}_{\beta \gamma c d}^{\alpha} Q_{c d}\left(\Gamma^{\beta} f, \Gamma^{\gamma} g\right)
\end{aligned}
$$

and similarly, 


$$
\Gamma^{\alpha} Q(f, g)=Q\left(\Gamma^{\alpha} f, g\right)+Q\left(f, \Gamma^{\alpha} g\right)+\sum_{|\beta|+|\gamma| \leq k-1} C_{\beta \gamma}^{\alpha} Q\left(\Gamma^{\beta} f, \Gamma^{\gamma} g\right) .
$$

Hence it suffices to prove the assertions for $k=0$. When $t \leq 1$, it is clear that (i ) and (ii) hold. From the definition of $\Gamma$ 's, we can write

$$
\begin{aligned}
Q_{12}(f, g) & =\frac{1}{t}\left(\Omega_{01} f \partial_{2} g-\Omega_{02} \partial_{1} g-\partial_{t} f \Omega_{12} g\right), \\
Q_{0 j}(f, g) & =\frac{1}{t}\left(\partial_{t} f \Omega_{0 j} g-\Omega_{0 j} f \partial_{t} g\right), \\
Q(f, g) & =\frac{1}{t}\left(\partial_{t} f \Gamma_{0} g-\sum_{j=1}^{2} \Omega_{0 j} \partial_{j} g\right) .
\end{aligned}
$$

Therefore the assertion holds for $t \geq 1$ and this completes the proof.

\section{§ 3. Preliminary Results for Linear Wave Equations}

In this section we state some results for linear wave equations

$$
\square u(t, x)=f(t, x), \quad(t, x) \in \overline{\boldsymbol{R}}_{+} \times \boldsymbol{R}^{2},
$$

where $f$ is a smooth function satisfying

$$
f(t, x)=0 \text { for }|x|>t+R
$$

with some constant $R>0$.

Lemma 3.1. Let $u(t, x)$ be a smooth solution of (3.1) with initial data 0 . Suppose $0 \leq \kappa \leq 1$. Then there exists a constant $C>0$ such that

$$
(1+t+|x|)^{1 / 2}(1+|t-| x||)^{(1-\kappa) / 2}|u(t, x)| \leq C \int_{0}^{t} \frac{\|f(s)\|_{1,1}}{(1+s)^{\kappa / 2}} d s .
$$

Proof. See Hörmander [4]. In fact, in Corollary 6.2 of [4], it was shown that

$$
\begin{aligned}
& \left\{\left(1+\left.\left|t^{2}-\right| x\right|^{2} \mid+\left(t^{2}+|x|^{2}\right)^{1 / 2}\right)^{1-\kappa}\left(1+\left(t^{2}+|x|^{2}\right)^{1 / 2}\right)^{\kappa}\right\}^{1 / 2}|u(t, x)| \\
& \leq C \sum_{|\alpha| \leq 1} \int_{0}^{t} \int_{R^{2}} \frac{\left|\Gamma^{\alpha} f(s, y)\right|}{\left\{1+\left(s^{2}+|y|^{2}\right)^{1 / 2}\right\}^{\kappa / 2}} d y d s .
\end{aligned}
$$

Observing that $(1+t+|x|)(1+|t-| x||) \leq 2 \sqrt{2}\left(1+\left.\left|t^{2}-\right| x\right|^{2} \mid+\left(t^{2}+|x|^{2}\right)^{1 / 2}\right)$, the assertion follows immediately. 
The next proposition is due to $\mathrm{Li}-\mathrm{Yu}-\mathrm{Zhou}[13]$.

Proposition 3. 2. Suppose u satisfies (3.1), then

$$
\begin{aligned}
& \text { (i ) }\|\partial u(t)\|_{2} \leq C\left(\|\partial u(0)\|_{2}+\int_{0}^{t}\|f(s)\|_{2} d s\right), \\
& \text { (ii) }\|u(t)\|_{2} \leq\|u(0)\|_{2}+C_{\delta} t^{2 \delta /(1+\delta)}\{\left\|\partial_{t} u(0)\right\|_{1+\delta} \\
&\left.+\int_{0}^{t}\|f(s)\|_{1+\delta} d s\right\}
\end{aligned}
$$

for $0<\delta<1$, where $C_{\delta}$ is a constant depending on $\delta$.

Proof. (i ) is a standard energy estimate. So we prove here only (ii). First, let $u$ be a solution of $\square u=0$ with initial data $u(0)=u_{0}$ and $\partial_{t} u(0)=$ $u_{1}$. Let $\mathscr{F}$ denote Fourier transform and $\hat{v}(\xi)=\mathscr{F}[v](\xi)$. Then it is well known that

$$
\hat{u}(t, \xi)=\hat{u}_{0}(\xi) \cos |\xi| t+\frac{\hat{u}_{1}(\xi)}{|\xi|} \sin |\xi| t
$$

Fix any $\rho$ satisfying $0<\rho<1$. We have

$$
\begin{aligned}
\left|\frac{\sin |\xi| t}{|\xi|} \hat{u}_{1}\right| & =\left|\frac{\sin |\xi| t}{|\xi|}\right|^{\rho}\left|\frac{(\sin |\xi| t)^{1-\rho} \hat{u}_{1}}{|\xi|^{1-\rho}}\right| \\
& \leq\left|\frac{\sin |\xi| t}{|\xi|}\right|^{\rho}\left|\frac{\hat{u}_{1}}{|\xi|^{1-\rho}}\right| \leq C_{\rho} t^{\rho}\left|\frac{\hat{u}_{1}}{|\xi|^{1-\rho}}\right| .
\end{aligned}
$$

Let $\phi_{a}(x)=|x|^{-2 / a}$ for $x \in \boldsymbol{R}^{2}$, then $\mathscr{F}\left[\psi_{a}\right](\xi)=C_{a}|\xi|^{-2+2 / a}$ for any $a>1$. (See Mizohata [11] for instance.) So it follows from Parseval's formula that

$$
\left\|\frac{\hat{u}_{1}}{|\xi|^{1-\rho}}\right\|_{2}=C_{\rho}\left\|\phi_{\frac{2}{(1+\rho)}} * u_{1}\right\|_{2} .
$$

Hardy-Littlewood inequality implies that

$$
\left\|\phi_{\frac{2}{(1+o)}} * u_{1}\right\|_{2} \leq C_{\rho}\left\|u_{1}\right\|_{p}
$$

where $1 / p=3 / 2-(1+\rho) / 2=(2-\rho) / 2$. (See Hörmander $[3 ;$ Theorem 4. 5. 3]. ) Therefore we get

$$
\|u(t, \cdot)\|_{2} \leq\left\|u_{0}\right\|_{2}+C_{\rho} t^{\rho}\left\|u_{1}\right\|_{1+\rho /(2-\rho)} .
$$

Choose $\rho=2 \delta /(1+\delta)(0<\delta<1)$, then we obtain

$$
\|u(t, \bullet)\|_{2} \leq\left\|u_{0}\right\|_{2}+C_{\delta} t^{2 \delta /(1+\delta)}\left\|u_{1}\right\|_{1+\delta}
$$


Now let $u$ be a solution of $\square u=f$ with initial data 0 . From Duhamel's principle we can write

$$
u(t, x)=\int_{0}^{t} U(t, x ; s) d s
$$

where $U(t, x ; s)$ is a solution of $\square U(t, x ; s)=0$ for $t \geq s$ with initial data $U(s, x ; s)=0$ and $\left(\partial_{t} U\right)(s, x ; s)=f(s, x)$. (3.6) leads to

$$
\|U(t, \bullet ; s)\|_{2} \leq C_{\delta}(t-s)^{2 \delta /(1+\delta)}\|f(s, \bullet)\|_{1+\delta}
$$

Thus it follows that

$$
\begin{aligned}
\|u(t)\|_{2} & =\left\|\int_{0}^{t} U(t, \bullet ; s) d s\right\|_{2} \leq \int_{0}^{t}\|U(t, \bullet ; s)\|_{2} d s \\
& \leq C_{\delta} t^{2 \delta /(1+\delta)} \int_{0}^{t}\|f(s, \bullet)\|_{1+\delta} d s .
\end{aligned}
$$

Combining (3.6) with (3.7), we obtain the result for the general case.

Remark 3. Proposition 3. 2 (ii) does not hold if we choose $\delta=0$. In fact, consider the Cauchy problem

$$
\square u(t, x)=0 \quad \text { in } \quad(0, \infty) \times \boldsymbol{R}^{2},
$$

with $u(0, x)=0, \partial_{t} u(0, x)=g(x)$. Suppose that $g \in C_{0}^{\infty}\left(\boldsymbol{R}^{2}\right), g \geq 0$ and $m=$ $\int_{R^{2}} g(x) d x>0$. Then $\hat{g}(0)=m>0$. So there exists some constant $A>0$ such that $|\hat{g}(\xi)| \geq m / 2$ for $|\xi|<A$. As $\hat{u}(t, \xi)=(\sin |\xi| t) \hat{g}(\xi) /|\xi|$, from Parseval's formula we get

$$
\begin{aligned}
\|u(t, \bullet)\|_{2}^{2} & =C\|\hat{u}(t, \bullet)\|_{2}^{2} \\
& =\int_{R^{2}} \frac{\sin ^{2}|\xi| t}{|\xi|^{2}}|\hat{g}(\xi)|^{2} d \xi \geq \frac{C m^{2}}{4} \int_{|\xi|<A} \frac{\sin ^{2}|\xi| t}{|\xi|^{2}} d \xi \\
& =\frac{\pi C m^{2}}{2} \int_{0}^{A} \frac{\sin ^{2} r t}{r} d r=\frac{\pi C m^{2}}{2} \int_{0}^{A t} \frac{\sin ^{2} \nu}{\nu} d \nu .
\end{aligned}
$$

Assume that there exists some constant $B>0$ such that $\left\|u\left(t,{ }^{\circ}\right)\right\|_{2} \leq B$ for $0 \leq t<\infty$. Then it follows that

$$
\int_{0}^{\infty} \frac{\sin ^{2} \nu}{\nu} d \nu \leq \frac{2 B^{2}}{\pi C m^{2}} .
$$

But as 


$$
\begin{aligned}
\int_{0}^{\infty} \frac{\sin ^{2} \nu}{\nu} d \nu & =\sum_{j=0}^{\infty} \int_{2 \pi j}^{2 \pi(j+1)} \frac{\sin ^{2} \nu}{\nu} d \nu \\
& \geq \int_{0}^{2 \pi} \sin ^{2} \nu d \nu \sum_{j=0}^{\infty} \frac{1}{2 \pi(j+1)}=\infty
\end{aligned}
$$

this is a contradiction. So $\|u(t, \bullet)\|_{2}$ cannot be bounded.

Lemma 3. 3. Let $u$ be a smooth solution of

$$
\square u(t, x)=\sum_{a=0}^{2} C_{a} \partial_{a} f(t, x), \quad(t, x) \in \overline{\boldsymbol{R}}_{+} \times \boldsymbol{R}^{2}
$$

with initial data 0. Then

$$
\|u(t)\|_{2} \leq C_{\delta} t^{2 \delta /(1+\delta)}\|f(0)\|_{1+\delta}+C \int_{0}^{t}\|f(s)\|_{2} d s
$$

for $0<\delta<1$

Proof. This is an analogue of the result of Lindblad [10]. (In fact his result concerns the case $n=3$, but there is no difference in the proof.)

Let $v$ be a solution of $\square v=f$ with initial data 0 , and let $w$ be a solution of $\square w=0$ with initial data $w(0, x)=0, \partial_{t} w(0, x)=f(0, x)$. Then $u$ can be written as $u=\sum_{a=0}^{2} C_{a} \partial_{a} v-C_{0} w$. So we obtain the result using Proposition 3. 2.

Proposition 3.4. Let $u(t, x)$ be a solution of (3.1) with initial data 0 . Then it follows that

$$
\|u(t)\|_{2,1} \leq C_{\delta} t^{2 \delta /(1+\delta)} \int_{0}^{t}\|f(s)\|_{1+\delta} d s+C \int_{0}^{t}(s+R)\|f(s)\|_{2} d s
$$

for $0<\delta<1$, where $R$ is the same constant as in (3.2).

Proof. From Proposition 3. 2 (ii), we get

$$
\|u(t)\|_{2} \leq C_{\delta} t^{2 \delta /(1+\delta)} \int_{0}^{t}\|f(s)\|_{1+\delta} d s
$$

From (2.2) it follows that

$$
\square\left(\Gamma_{0} u\right)=\left(\Gamma_{0}+2\right) f=\partial_{t}(t f)+\sum_{i=1}^{2} \partial_{i}\left(x_{i} f\right)-f .
$$

As $\left(\Gamma_{0} u\right)(0)=\left(\partial_{t} \Gamma_{0} u\right)(0)=0$, noting that $|x| \leq t+R$ in supp $f$, Proposition 3. 2 and Lemma 3.3 imply that 


$$
\begin{aligned}
&\left\|\Gamma_{0} u(t)\right\|_{2} \leq C \int_{0}^{t}\left\{\|s f(s)\|_{2}+\sum_{i=1}^{2}\left\|\left(x_{i} f\right)(s)\right\|_{2}\right\} d s \\
&+C_{\delta} t^{2 \delta /(1+\delta)} \int_{0}^{t}\|f(s)\|_{1+\delta} d s \\
& \leq C \int_{0}^{t}(s+R)\|f(s)\|_{2} d s+C_{\delta} t^{2 \delta /(1+\delta)} \int_{0}^{t}\|f(s)\|_{1+\delta} d s .
\end{aligned}
$$

For $i=1,2$, it follows that

$$
\begin{gathered}
\square\left(\Omega_{0 i} u\right)=\Omega_{0 i} f=\partial_{i}(t f)+\partial_{t}\left(x_{i} f\right), \\
\left(\Omega_{0 i} u\right)(0)=0,\left(\partial_{t} \Omega_{0 i} u\right)(0)=x_{i} f(0, x)
\end{gathered}
$$

As in the proof of Lemma 3. 3, we obtain

$$
\begin{aligned}
\left\|\Omega_{0 i} u(t)\right\|_{2} & \leq C \int_{0}^{t}\left\{\|s f(s)\|_{2}+\left\|\left(x_{i} f\right)(s)\right\|_{2}\right\} d s \\
& \leq C \int_{0}^{t}(s+R)\|f(s)\|_{2} d s .
\end{aligned}
$$

Similarly, we get from Lemma 3. 3 that

$$
\begin{aligned}
\left\|\Omega_{12} u(t)\right\|_{2} & \leq C \int_{0}^{t}\left\{\left\|\left(x_{2} f\right)(s)\right\|_{2}+\left\|\left(x_{1} f\right)(s)\right\|_{2}\right\} d s \\
& \leq C \int_{0}^{t}(s+R)\|f(s)\|_{2} d s .
\end{aligned}
$$

Finally, $\|\partial u(t)\|_{2} \leq C \int_{0}^{t}\|f(s)\|_{2} d s$ from Proposition 3.2(i ) and this completes the proof.

Lemma 3. 5. Let $u \in C^{\infty}\left(\overline{\boldsymbol{R}}_{+} \times \boldsymbol{R}^{2}\right)$ be a solution of

$$
\square u(t, x)-\sum_{a, b} \gamma_{a b}(t, x) \partial_{a} \partial_{b} u(t, x)=f(t, x)
$$

Suppose that

$$
\sum_{a, b}\left|\gamma_{a b}(t, x)\right| \leq \frac{1}{2}
$$

for all $(t, x) \in \overline{\boldsymbol{R}}_{+} \times \boldsymbol{R}^{2}$. Define

$$
\|u(t)\|_{E}^{2}=\int_{R^{2}}\left\{\beta_{00}\left|\partial_{t} u\right|^{2}+\sum_{h j=1}^{2} \beta_{i j} \partial_{i} u \partial_{j} u\right\} d x
$$

where $\beta_{00}=1-\gamma_{00}, \beta_{i j}=\delta_{i j}+\gamma_{i j}$ for any $i, j=1,2$. Then there exist some constants $c, C>0$ such that 
(3. 10)

$$
\frac{1}{c}\|u(t)\|_{E}^{2} \leq\|\partial u(t)\|_{2}^{2} \leq c\|u(t)\|_{E}^{2}
$$

and

$$
\frac{d}{d t}\|u(t)\|_{E} \leq C\left\|\gamma^{\prime}(t)\right\|_{\infty}\|u(t)\|_{E}+\|f(t)\|_{2},
$$

where $\left\|\gamma^{\prime}(t)\right\|_{\infty}=\max _{a, b, c}\left\|\partial_{c} \gamma_{a b}(t, \bullet)\right\|_{\infty}$.

Proof. See Klainerman [6].

To conclude this section, we state two technical lemmata to be used in the proof of Theorem 1 .

Lemma 3. 6. Let

$$
\phi(t, x)=(1+t+|\boldsymbol{x}|)^{-\alpha / 2}(1+|t-| \boldsymbol{x}||)^{-\alpha(1-\kappa) / 2}, 0<\kappa<1 .
$$

If $\alpha p(1-\kappa)>2$, then

$$
\|\phi(t, \cdot)\|_{p} \leq C_{\alpha, \kappa, p}(1+t)^{-\alpha / 2+1 / p}
$$

Proof.

$$
\|\phi(t, \cdot)\|_{p}^{p} \leq(1+t)^{-\alpha p \kappa / 2} \int_{R^{2}}\{(1+t+|x|)(1+|t-| x||)\}^{-\alpha p(1-\kappa) / 2} d x .
$$

Switching to the polar coordinate, we get

$$
\begin{aligned}
\int_{R^{2}} & \{(1+t+|x|)(1+|t-| x||)\}^{-\alpha p(1-\kappa) / 2} d x \\
& =2 \pi \int_{0}^{\infty}\{(1+t+r)(1+|t-r|)\}^{-\alpha p(1-\kappa) / 2} r d r \\
& =2 \pi\left\{\int_{0}^{t}\left\{(1+t)^{2}-r^{2}\right\}^{-\alpha p(1-\kappa) / 2} r d r+\int_{t}^{\infty}\left\{(1+r)^{2}-t^{2}\right\}^{-\alpha p(1-\kappa) / 2} r d r\right\} \\
& \leq \frac{2 \pi}{\alpha p(1-\kappa)-2}\left(\left[\left\{(1+t)^{2}-r^{2}\right\}^{1-\alpha p(1-\kappa) / 2}\right]_{r=0}^{t}\right. \\
& \left.\quad+\left[-\left\{(1+r)^{2}-t^{2}\right\}^{1-\alpha p(1-\kappa) / 2}\right]_{r=t}^{\infty}\right) \\
& \left.=\frac{2 \pi}{\alpha p(1-\kappa)-2}\left\{(1+2 t)^{1-\alpha p(1-\kappa) / 2}-(1+t)^{2-\alpha p(1-\kappa)}\right]^{1-\alpha p(1-\kappa) / 2}\right\} \\
\leq & C_{\alpha, \kappa, p}(1+t)^{-\alpha p(1-\kappa) / 2+1} .
\end{aligned}
$$


This completes the proof.

Lemma 3. 7. Suppose $v, w$ be smooth functions in $\overline{\boldsymbol{R}}_{+} \times \boldsymbol{R}^{2}$, and

$$
\operatorname{supp} w \subset\{|x| \leq t+R\}
$$

with some constant $R>0$. Then

$$
\left\|\left(\partial_{a} v \circ w\right)(t, \circ)\right\|_{2} \leq C\|v(t, \bullet)\|_{\infty, 1}\|\partial w(t, \bullet)\|_{2}
$$

for $0 \leq a \leq 2$.

Proof. First one can verify that

$$
(1+|t-| x||)^{2}|(\partial v)(t, x)|^{2} \leq 4|v(t, x)|_{1}^{2}
$$

and

$$
\left\|\frac{w(t, \bullet)}{1+|t-| \cdot||}\right\|_{2} \leq C\|\partial w(t, \bullet)\|_{2}
$$

Therefore we get

$$
\begin{aligned}
\left\|\left(\partial_{a} v \cdot w\right)(t, \bullet)\right\|_{2} & \leq C\left\|\left.v(t, \bullet)\right|_{1} \frac{w(t, \bullet)}{1+|t-| \cdot||}\right\|_{2} \\
& \leq C\|v(t, \bullet)\|_{\infty, 1}\left\|\frac{w(t, \bullet)}{1+|t-| \cdot||}\right\|_{2} \\
& \leq C\|v(t, \bullet)\|_{\infty, 1}\|\partial w(t, \bullet)\|_{2}
\end{aligned}
$$

See Lindblad [10] and Godin [2] for details.

\section{$\S 4 . \quad$ Proof of Theorem 1}

In this section we prove Theorem 1 . First we make some a priori estimates. Let $u$ be a solution of (1.1)-(1.2) for $0 \leq t<T$. Fix some integer $k \geq 4$. Define

(4. 1) $\quad M_{1}(t ; u)=\sup _{0 \leq s<t} \sup _{y \in R^{2}}(1+s+|y|)^{1 / 2}(1+|s-| y||)^{1 / 4}|u(s, y)|_{k+2}$,

(4. 2) $\quad M_{2}(t ; u)=\sup _{0 \leq s<t}(1+s)^{-\lambda}\|u(s, \bullet)\|_{2,2 k}$,

(4. 3) $\quad M_{3}(t ; u)=\sup _{0 \leq s<t}(1+s)^{-\mu}\|\partial u(s, \bullet)\|_{2,2 k}$ 
for $0 \leq t<T$ with $\lambda=1 / 10, \mu=1 / 30$, say. We show that there exist some constants $\varepsilon_{0}>0$ and $M>0$ (which are independent of $T$ ), such that if $\varepsilon<\varepsilon_{0}$, then $\max _{i=1,2,3} M_{i}(t ; u) \leq M \varepsilon$ holds for the solution $u(t, x)$ of (1.1)-(1.2) on $t \in[0, T)$. Combining this a priori estimate with the classical local existence theorem, we can prove the theorem.

Let

$$
\tau=\sup \left\{0 \leq t<T ; \max _{i=1,2,3} M_{i}(s ; u) \leq M \varepsilon \text { for } 0 \leq s<t\right\}
$$

In the following, we are going to show that $\tau=T$ for any $\varepsilon \leq \varepsilon_{0}$, if $M$ is sufficiently large and $\varepsilon_{0}$ is sufficiently small, and this proves the above a priori estimate.

The local existence theorem implies that $\tau>0$, if $M$ is chosen appropriately large Let $\varepsilon$ be so small that $M \varepsilon<1$.

\section{§4. 1. $L^{\infty}$-estimates}

For $|\alpha| \leq k+2$, it follows that

$$
\square\left(\Gamma^{\alpha} u_{i}\right)=\sum_{|\beta| \leq k+2} C_{\beta}^{\alpha}\left(\Gamma^{\beta} G_{i}+\Gamma^{\beta} H_{i}\right), \quad i=1, \cdots, N .
$$

Lemma 2. 2 implies that

$$
\left\|\Gamma^{\beta} G_{i}\right\|_{1,1} \leq C(1+s)^{-1}\|u(s)\|_{\infty,[(k+3) / 2]+2}
$$

$$
\begin{gathered}
\cdot\left\{\|u(s)\|_{2, k+4}+\|\partial u(s)\|_{2, k+4}\right\}^{2} \\
\leq C(1+s)^{2 \lambda-3 / 2} M^{3} \varepsilon^{3}, \quad 0 \leq s<\tau
\end{gathered}
$$

for $|\beta| \leq k+2$, because $[(k+3) / 2]+2 \leq k+2$ and $k+4 \leq 2 k$.

As $H=O\left(|u|^{4}+\left|u^{\prime}\right|^{4}+\left|u^{\prime \prime}\right|^{4}\right)$, we get

$$
\left\|\Gamma^{\beta} H_{i}\right\|_{1,1} \leq C\left\|\left.u(s, \bullet)\right|_{[(k+3) / 2]+2} ^{3}\right\|_{2}\left(\|u(s, \bullet)\|_{2, k+4}+\|\partial u(s, \bullet)\|_{2, k+4}\right) .
$$

(4. 4) and Lemma 3.6 imply that

$$
\text { (4. 7) } \begin{aligned}
\left\||u(s, \cdot)|_{[(k+3) / 2]+2}^{3}\right\|_{2} & \leq C M^{3} \varepsilon^{3}\left\|(1+s+|\cdot|)^{-3 / 2}(1+|s-| \cdot||)^{-3 / 4}\right\|_{2} \\
& \leq C M^{3} \varepsilon^{3}(1+s)^{-1}
\end{aligned}
$$

for $0 \leq s<\tau$. Therefore we get 


$$
\left\|\Gamma^{\beta} H_{i}\right\|_{1,1} \leq C M^{4} \varepsilon^{4}(1+s)^{\lambda-1} \text { for } 0 \leq s<\tau .
$$

Let $\tilde{u}_{i}^{\alpha}(t, x)$ be a solution of $\square \tilde{u}_{i}^{\alpha}=0$ with initial data $\tilde{u}_{i}^{\alpha}(0)=\left(\Gamma^{\alpha} u_{i}\right)(0)$ and $\left(\partial_{t} \tilde{u}_{i}^{\alpha}\right)(0)=\left(\partial_{t} \Gamma^{\alpha} u_{i}\right)(0)$. From the well-known decay estimate we can show that

$$
(1+t+|\boldsymbol{x}|)^{1 / 2}(1+|t-| \boldsymbol{x}||)^{1 / 2}\left|\tilde{u}_{i}^{\alpha}(t, x)\right| \leq C_{k} \varepsilon
$$

where $C$ is a positive constant which may depend on $k, f$ and $g$.

From (4. 5), (4.6), (4. 8), (4.9) and Lemma 3. 1 with $\kappa=1 / 2$, it follows that

$$
\begin{aligned}
(1+t+ & |\boldsymbol{x}|)^{1 / 2}(1+|t-| \boldsymbol{x}||)^{1 / 4}\left|\Gamma^{\alpha} u_{i}(t, x)\right| \\
& \leq C\left(\varepsilon+M^{3} \varepsilon^{3} \int_{0}^{t}(1+s)^{-1 / 4}\left\{(1+s)^{2 \lambda-3 / 2}+(1+s)^{\lambda-1} M \varepsilon\right\} d s\right. \\
& \leq C\left(1+M^{3} \varepsilon^{2}\right) \varepsilon
\end{aligned}
$$

for $|\alpha| \leq k+2$, because $2 \lambda-7 / 4<-1$ and $\lambda-5 / 4<-1$. So we obtain

$$
M_{1}(\mathrm{t} ; u) \leq C\left(1+M^{3} \varepsilon^{2}\right) \varepsilon \quad \text { for } 0 \leq t<\tau
$$

where $C$ is a constant independent of $M$ and $\varepsilon$.

\section{§ 4. 2. $L^{2}$-estimates}

For $|\alpha| \leq 2 k-1$, we get as before

$$
\square\left(\Gamma^{\alpha} u_{i}\right)=\sum_{|\beta| \leq 2 k-1} C_{\beta}^{\alpha} \Gamma^{\beta} G_{i}+\sum_{|\beta| \leq 2 k-1} C_{\beta}^{\alpha} \Gamma^{\beta} H_{i}, \quad i=1, \cdots, N .
$$

Write $H_{i}=I_{i}+J_{i}, i=1, \cdots, N$, where $I_{i}\left(u, u^{\prime}, u^{\prime \prime}\right)$ is a homogeneous polynomial of degree four, and $J_{i}=O\left(|\boldsymbol{u}|^{5}+\left|\boldsymbol{u}^{\prime}\right|^{5}+\left|\boldsymbol{u}^{\prime \prime}\right|^{5}\right)$.

Let $v_{i}^{\alpha}$ be a solution of $\square v_{i}^{\alpha}=\sum_{|\beta| \leq 2 k-1} C_{\beta}^{\alpha} \Gamma^{\beta}\left(G_{i}+J_{i}\right)$ with initial data 0 , $w_{i}^{\alpha}$ be a solution of $\square w_{i}^{\alpha}=\sum_{|\beta| \leq 2 k-1} C_{\beta}^{\alpha} \Gamma^{\beta} I_{i}$ with $\left(\partial_{t}^{m} w_{i}^{\alpha}\right)(0)=\left(\partial_{t}^{m} \Gamma^{\alpha} u_{i}\right)(0)$ for $m=0,1$. Then $\Gamma^{\alpha} u_{i}=v_{i}^{\alpha}+w_{i}^{\alpha}$.

Lemma 2. 2 implies that

$$
\begin{aligned}
& \left\|\Gamma^{\beta} \boldsymbol{G}_{i}\right\|_{2} \\
& \quad \leq C(1+s)^{-1}\|u(s, \cdot)\|_{\infty, k+2}^{2}\left\{\|u(s, \cdot)\|_{2,2 k}+\|\partial u(s, \bullet)\|_{2,2 k}\right\} \\
& \quad \leq C(1+s)^{\lambda-2} M^{3} \varepsilon^{3}
\end{aligned}
$$


and for $0<\delta<1$,

$$
\begin{array}{r}
\left\|\Gamma^{\beta} \boldsymbol{G}_{i}\right\|_{1+\delta} \leq(1+s)^{-1}\left\|\left.\boldsymbol{u}(s, \bullet)\right|_{k+2} ^{2}\right\|_{2(1+\delta) /(1-\delta)}\left\{\|\boldsymbol{u}(s, \bullet)\|_{2,2 k}\right. \\
\left.+\|\partial u(s, \bullet)\|_{2,2 k}\right\}
\end{array}
$$

$$
\leq C(1+s)^{\lambda-1} M \varepsilon\left\||u(s, \cdot)|_{k+2}^{2}\right\|_{2(1+\delta) /(1-\delta)}
$$

for $|\beta| \leq 2 k-1$. As $J_{i}=O\left(|u|^{5}+\left|u^{\prime}\right|^{5}+\left|u^{\prime \prime}\right|^{5}\right)$, we have

(4. 13) $\quad\left\|\Gamma^{\beta} J_{i}\right\|_{2}$

$$
\begin{aligned}
& \leq \boldsymbol{C}\|\boldsymbol{u}(s, \cdot)\|_{\infty,[(2 k-1) / 2]+2}^{4}\left\{\|u(s, \cdot)\|_{2,2 k-1}+\|\partial u(s, \cdot)\|_{2,2 k-1}\right\} \\
& \leq C(1+s)^{\lambda-2} M^{5} \varepsilon^{5} \leq C(1+s)^{\lambda-2} M^{3} \varepsilon^{3}
\end{aligned}
$$

and

$$
\left\|\Gamma^{\beta} J_{i}\right\|_{1+\delta} \leq C\|u(s, \cdot)\|_{\infty,[(2 k-1) / 2]+2}^{2}\left\||u(s, \cdot)|_{[(2 k-1) / 2]+2}^{2}\right\|_{2(1+\delta) /(1-\delta)}
$$

$$
\text { - }\left\{\|u(s, \bullet)\|_{2,2 k-1}+\|\partial u(s, \cdot)\|_{2,2 k-1}\right\}
$$

$$
\leq C(1+s)^{\lambda-1} M^{3} \varepsilon^{3}\left\||u(s, \cdot)|_{k+2}^{2}\right\|_{2(1+\delta) /(1-\delta)}
$$

for $|\beta| \leq 2 k-1$, as $M \varepsilon \leq 1$. Because $2(1+\delta) /(1-\delta)>2$, we can use Lemma 3. 6 to get

$$
\left.\|\| \boldsymbol{u}(s, \cdot)\right|_{k+2} ^{2} \|_{2(1+\delta) /(1-\delta)} \leq C_{\delta} M^{2} \varepsilon^{2}(1+s)^{-1+(1-\delta) / 2(1+\delta)}
$$

for $0 \leq s<\tau$. Therefore Proposition 3. 2 (ii) and Proposition 3. 4 imply that

$$
\begin{aligned}
\left\|v_{i}^{\alpha}(t)\right\|_{2,1} \leq C \int_{0}^{t}(1+s)^{\lambda-1} M^{3} \varepsilon^{3} d s & \quad+C_{\delta}(1+t)^{2 \delta /(1+\delta)} \int_{0}^{t}(1+s)^{\lambda-2+\frac{1-\delta}{2(1+\delta)}} M^{3} \varepsilon^{3} d s \\
& \leq C_{\delta}(1+t)^{\lambda} M^{3} \varepsilon^{3}
\end{aligned}
$$

for $|\alpha| \leq 2 k-1$, provided that $2 \delta /(1+\delta) \leq \lambda$ and $\lambda-2+\frac{1-\delta}{2(1+\delta)}<-1$. This holds if we choose $\delta=1 / 20$, say.

Now we are going to estimate $\left\|w_{i}^{\alpha}(t)\right\|_{2,1}$. As $F(u, 0,0)=O\left(|u|^{5}\right)$, it follows that $I_{i}(u, 0,0)=0, \quad i=1, \cdots, N$, so we can write 


$$
I_{i}\left(u, u^{\prime}, u^{\prime \prime}\right)=\sum_{a, b} I_{i}^{a b}\left(u, u^{\prime}\right) \partial_{a} \partial_{b} u_{i}+\sum_{a, j} I_{i}^{a, j}\left(u, u^{\prime}\right) \partial_{a} u_{j} .
$$

For $|\gamma| \leq 1$, we have

$$
\begin{aligned}
\square\left(\Gamma^{\gamma} \boldsymbol{w}_{i}^{\alpha}\right) & =\sum_{|\beta| \leq 2 k} C_{\beta}^{\alpha, \gamma} \Gamma^{\beta} I_{i} \\
& =\sum_{|\beta|=2 k} C_{\beta}^{\alpha, \gamma} \sum_{a, b} \partial_{a}\left\{I_{i}^{a b} \cdot \Gamma^{\beta} \partial_{b} u_{i}\right\}+R_{i}^{\alpha, \gamma},
\end{aligned}
$$

where

$$
\begin{aligned}
R_{i}^{\alpha, \gamma}=\sum_{|\beta| \leq 2 k} C_{\beta}^{\alpha, \gamma} & \Gamma^{\beta} I_{i}-\sum_{|\beta|=2 k} C_{\beta}^{\alpha, \gamma} \sum_{a, b} I_{i}^{a b} \cdot \Gamma^{\beta}\left(\partial_{a} \partial_{b} u_{i}\right) \\
& +\sum_{|\beta|=2 k} C_{\beta}^{\alpha, \gamma} \sum_{a, b} I_{i}^{a b}\left[\Gamma^{\beta}, \partial_{a}\right] \partial_{b} u_{i} \\
& -\sum_{\mid \beta=2 k} C_{\beta}^{\alpha, \gamma} \sum_{a, b}\left(\partial_{a} I_{i}^{a b}\right) \cdot \Gamma^{\beta} \partial_{b} u_{i} .
\end{aligned}
$$

First it is easily seen that

$$
\begin{aligned}
\left\|I_{i}^{a b} \cdot \Gamma^{\beta} \partial_{b} u_{i}\right\|_{2} & \leq C\|u(s, \cdot)\|_{\infty, 1}^{3}\|\partial u(s, \cdot)\|_{2,2 k} \\
& \leq C(1+s)^{\mu-3 / 2} M^{4} \varepsilon^{4} \text { for }|\beta|=2 k .
\end{aligned}
$$

$R_{i}^{\alpha, \gamma}$ is a linear combination of terms of the form

$$
\Gamma^{\gamma_{1}} \partial^{\nu_{1}} u_{j_{1}} \Gamma^{\gamma_{2}} \partial^{\nu_{2}} u_{j_{2}} \Gamma^{\gamma_{3}} \partial^{\nu_{3}} u_{j_{3}} \Gamma^{\gamma_{4}} \partial^{\nu_{4}} u_{j_{4}}
$$

with $j_{l} \in\{1, \cdots, N\}, 0 \leq\left|\nu_{1}\right|,\left|\nu_{2}\right|,\left|\nu_{3}\right| \leq 2,1 \leq\left|\nu_{4}\right| \leq 2,\left|\gamma_{1}\right|,\left|\gamma_{2}\right| \leq k$, $\sum_{l=1}^{4}\left|\gamma_{l}\right| \leq 2 k$ and $\left|\gamma_{l}\right|+\left|\nu_{l}\right| \leq 2 k+1$ for $l=1, \cdots, 4$. Here $\partial^{\nu} l$ denotes $\partial_{0}^{\nu l, 0} \partial_{1}^{\nu_{l, 1}} \partial_{2}^{\nu l, 2}$ for $\nu_{l}=\left(\nu_{l, 0}, \nu_{l, 1}, \nu_{l, 2}\right)$.

When $\left|\gamma_{4}\right| \geq\left|\gamma_{3}\right|$, Hörder's inequality implies that

$$
\begin{aligned}
\left\|\prod_{l=1}^{4} \Gamma^{\gamma_{l}} \partial^{\nu}{ }_{l} u_{j_{l}}\right\|_{1+\delta} & \leq\left\|\Gamma^{\gamma_{1}} \partial^{\nu_{1}} u_{j_{1}} \Gamma^{\gamma_{2}} \partial^{\nu_{2}} u_{j_{2}}\right\|_{2(1+\delta) /(1-\delta)} \bullet\left\|\Gamma^{\gamma_{3}} \partial^{\nu_{3}} u_{j_{3}} \cdot \Gamma^{\gamma_{4}} \partial^{\nu_{4}} u_{j_{4}}\right\|_{2} \\
& \leq\left\|\left.\boldsymbol{u}(t, \bullet)\right|_{k+2} ^{2}\right\|_{2(1+\delta) /(1-\delta)}\|u(t, \bullet)\|_{\infty, k+2}\|\partial u(t, \bullet)\|_{2,2 k}
\end{aligned}
$$

for any $0<\delta<1$. When $\left|\gamma_{4}\right|<\left|\gamma_{3}\right|$ and $\left|\nu_{3}\right| \geq 1$, we can estimate the term in the same way as above.

When $\left|\gamma_{4}\right|<\left|\gamma_{3}\right|$ and $\left|\nu_{3}\right|=0$, we obtain with the help of Lemma 3.7 and (2.8) that

$\left\|\prod_{l=1}^{4} \Gamma^{\gamma_{l}} \partial^{\nu}{ }^{\nu} u_{j_{l}}\right\|_{1+\delta} \leq\left\|\Gamma^{\gamma_{1}} \partial^{\nu_{1}} u_{j_{1}} \Gamma^{\gamma_{2}} \partial^{\nu_{2}} u_{j_{2}}\right\|_{2(1+\delta) /(1-\delta)}\left\|\Gamma^{\gamma_{3}} u_{j_{3}} \cdot \Gamma^{\gamma_{4}} \partial^{\nu_{4}} u_{j_{4}}\right\|_{2}$ 


$$
\begin{aligned}
& \leq \boldsymbol{C}|| \boldsymbol{u}(t, \cdot)||_{k+2}^{2}\left\|_{2(1+\delta) /(1-\delta)}\right\| u_{j_{4}}(t, \cdot)\left\|_{\infty, k+2}\right\| \partial\left(\Gamma^{\gamma_{3}} u_{j_{3}}\right) \|_{2} \\
& \leq\left\|\boldsymbol{u}(t, \cdot) \mid{ }_{k+2}^{2}\right\|_{2(1+\delta) /(1-\delta)}\|\boldsymbol{u}(t, \cdot)\|_{\infty, k+2}\|\partial u(t, \cdot)\|_{2,2 k} .
\end{aligned}
$$

Therefore

$$
\left\|R_{i}^{\alpha, r}\right\|_{1+\delta} \leq C M^{2} \varepsilon^{2}(1+t)^{\mu-1 / 2}\left\||u(t, \cdot)|_{k+2}^{2}\right\|_{2(1+\delta) /(1-\delta)} .
$$

As $2 \cdot 2(1+\delta) /(1-\delta) \cdot(1 / 2)>2$ for any $0<\delta<1$, Lemma 3.6 implies

$$
\begin{aligned}
\| \mid u(t, \cdot) & \left.\right|_{k+2} ^{2} \|_{2(1+\delta) /(1-\delta)} \\
& \leq M^{2} \varepsilon^{2}\left\|(1+t+|\cdot|)^{-1}(1+|t-| \cdot||)^{-1 / 2}\right\|_{2(1+\delta) /(1-\delta)} \\
& \leq C(1+t)^{-1+\frac{1-\delta}{2(1+\delta)}} M^{2} \varepsilon^{2} .
\end{aligned}
$$

So, from Proposition 3. 2 and Lemma 3. 3, it follows that

$$
\begin{gathered}
\left\|w_{i}^{\alpha}\right\|_{2,1} \leq C\left\{(1+t)^{2 \delta /(1+\delta)} \varepsilon+(1+t)^{2 \delta /(1+\delta)} M^{4} \varepsilon^{4} \int_{0}^{t}(1+s)^{\mu-\frac{3}{2}+\frac{(1-\delta)}{2(1+\delta)}} d s\right. \\
\left.+M^{4} \varepsilon^{4} \int_{0}^{t}(1+s)^{\mu-3 / 2} d s\right\} \\
\leq C(1+t)^{\lambda}\left(1+M^{4} \varepsilon^{3}\right) \varepsilon \quad \text { for } 0 \leq t<\tau,
\end{gathered}
$$

provided that $\mu+\frac{1-\delta}{2(1+\delta)}-\frac{3}{2}<-1$ and $\lambda \geq 2 \delta /(1+\delta)$. This holds if we choose $\delta=1 / 20$.

Finally, combining above estimates, we conclude that

$$
\|u(t, \cdot)\|_{2,2 k} \leq C(1+t)^{\lambda}\left(1+M^{3} \varepsilon^{2}\right) \varepsilon
$$

for $0 \leq t<\tau$, that is,

$$
M_{2}(t ; u) \leq C\left(1+M^{3} \varepsilon^{2}\right) \varepsilon \quad \text { for } 0 \leq t<\tau
$$

\section{§ 4. 3. The Energy Estimates}

Finally we make estimates for $L^{2}$-norms of the derivatives of the solution.

For $|\alpha| \leq 2 k$, we can write

$$
\begin{aligned}
& \square\left(\Gamma^{\alpha} u_{i}\right)-\sum_{a, b=0}^{2} F_{i}^{a b}\left(u, u^{\prime}\right) \cdot \partial_{a} \partial_{b}\left(\Gamma^{\alpha} u_{i}\right) \\
& \quad=\sum_{|\beta| \leq 2 k} C_{\beta}^{\alpha} \Gamma^{\beta} F_{i}-\sum_{a, b=0}^{2} F_{i}\left(u, u^{\prime}\right) \cdot \Gamma^{\alpha}\left(\partial_{a} \partial_{b} u_{i}\right)
\end{aligned}
$$




$$
+\sum_{a, b=0}^{2} F_{i}^{a b}\left(u, u^{\prime}\right)\left[\Gamma^{\alpha}, \partial_{a} \partial_{b}\right] u_{i},
$$

where $F_{i}^{a b}=\partial F_{i} / \partial u_{i, a b}$. Note that $C_{\beta}^{\alpha}=1$ if $\beta=\alpha$, and $C_{\beta}^{\alpha}=0$ if $|\beta|=2 k$ and $\beta \neq \alpha$. Because $F=O\left(|u|^{3}+\left|u^{\prime}\right|^{3}+\left|u^{\prime \prime}\right|^{3}\right)$ and $F(u, 0,0)=O\left(|u|^{5}\right)$, $L^{2}$-norms of the right-hand side of (4. 23) is bounded by

$$
\begin{gathered}
\|u(t, \bullet)\|_{\infty, k+2}^{2}\left(1+\|u(t, \cdot)\|_{\infty, k+2}\right)\|\partial u(t, \bullet)\|_{2,2 k} \\
\quad+\|u(t, \cdot)\| \|_{\infty, k+2}^{4}\left\{\|u(t, \bullet)\|_{2,2 k}+\|\partial u(t, \cdot)\|_{2,2 k}\right\} \\
\leq C(1+t)^{\mu-1} M^{3} \varepsilon^{3}+C(1+t)^{\lambda-2} M^{5} \varepsilon^{5}
\end{gathered}
$$

with the help of Lemma 3. 7.

As $\sup _{a, b, c}\left|\partial_{c} F_{i}^{a b}\left(u, u^{\prime}\right)\right| \leq|u(t, x)|_{2}^{2} \leq C(1+t)^{-1} M^{2} \varepsilon^{2}$ and $\sum_{a, b} \mid F_{i}^{a b}(u$, $\left.u^{\prime}\right) \mid \leq C(1+t)^{-1} M^{2} \varepsilon^{2}$, we can apply Lemma 3.5 to (4.11) if $\varepsilon$ is sufficiently small, and we obtain

$$
\frac{d}{d t}\left\|\Gamma^{\alpha} u(t)\right\|_{E} \leq C\|u(t, \bullet)\|_{\infty, 2}^{2}\left\|\Gamma^{\alpha} u(t, \bullet)\right\|_{E}
$$

$$
\begin{aligned}
& +C\|u(t, \bullet)\|_{\infty, k+2}^{2}\left(1+\|u(t, \bullet)\|_{\infty, k+2}\right)\|\partial u(t, \bullet)\|_{2,2 k} \\
& +C\|u(t, \bullet)\|_{\infty, k+2}^{4}\left\{\|u(t, \bullet)\|_{2,2 k}+\|\partial u(t, \bullet)\|_{2,2 k}\right\} \\
& \leq C(1+t)^{\mu-1} M^{3} \varepsilon^{3}
\end{aligned}
$$

for $|\alpha| \leq 2 k$, because $\|u(t)\|_{E} \leq C\|\partial u(t)\|_{2,2 k}$. This leads to

$$
\left\|\Gamma^{\alpha} u(t)\right\|_{E} \leq C\left(1+M^{3} \varepsilon^{2}\right)(1+t)^{\mu} \varepsilon \quad \text { for }|\alpha| \leq 2 k \text {. }
$$

As $\frac{1}{c}\left\|\partial \Gamma^{\alpha} u(t)\right\|_{2} \leq\left\|\Gamma^{\alpha} u(t)\right\|_{E}$, we have

$$
\left\|\partial\left(\Gamma^{\alpha} u\right)(t)\right\|_{2} \leq C\left(1+M^{3} \varepsilon^{2}\right)(1+t)^{\mu} \varepsilon \quad \text { for }|\alpha| \leq 2 k .
$$

In view of (2.8), this means that

$$
\|\partial u(t)\|_{2,2 k} \leq C\left(1+M^{3} \varepsilon^{2}\right)(1+t)^{\mu} \varepsilon \quad \text { for } 0 \leq t<\tau \text {, }
$$

i. e.,

$$
M_{3}(t ; u) \leq C\left(1+M^{3} \varepsilon^{2}\right) \varepsilon \quad \text { for } 0 \leq t<\tau .
$$




\section{§ 4. 4. Completion of the Proof of A Priori Estimates}

We have proved in sections 4. 1-4. 3 that if $u(t, x)$ is a solution to (1. 1)(1.2) for $0 \leq t<T$, and

$$
\tau=\sup \left\{0 \leq t<T ; \max _{i=1,2,3} M_{i}(s ; u) \leq M \varepsilon \text { for } 0 \leq s<t\right\}
$$

then

$$
\max _{i=1,2,3} M_{i}(t ; u) \leq C\left(1+M^{3} \varepsilon^{2}\right) \varepsilon \quad \text { for } 0 \leq t<\tau,
$$

where $C>0$ is a constant independent of $\varepsilon, \tau, T$ and $M$. Choose $M$ and $\varepsilon_{0}$ to satisfy $M \geq 2 C$ and $M^{3} \varepsilon_{0}^{2} \leq 1 / 2$, then from (4. 27) it follows that

$$
\max _{i=1,2,3} M_{i}(t ; u) \leq \frac{3}{2} C \varepsilon \leq \frac{3}{4} M \varepsilon, 0 \leq t<\tau
$$

for any $\varepsilon \leq \varepsilon_{0}$. By usual continuation arguments, we conclude that $\tau=T$. This completes the proof.

\section{§ 5. Some Remarks for the Single and Semilinear Equations}

In this section we consider the Cauchy problem for semilinear wave equations of the type

$$
\begin{gathered}
\square u=F\left(u, u^{\prime}\right) \quad \text { in } \overline{\boldsymbol{R}}_{+} \times \boldsymbol{R}^{2}, \\
u(0, x)=\varepsilon f(x), \partial_{t} u(0, x)=\varepsilon g(x), \quad x \in \boldsymbol{R}^{2},
\end{gathered}
$$

where $u$ and $F$ are scalar-valued functions and $f, g \in C_{0}^{\infty}\left(R^{2}\right)$.

Assume $F$ satisfies the following :

(A7) $\quad F(u, 0)=O\left(|u|^{5}\right)$,

(A8) $\quad F$ can be written as

$$
F\left(u, u^{\prime}\right)=G_{2}\left(u, u^{\prime}\right)+G_{3}\left(u, u^{\prime}\right)+H\left(u, u^{\prime}\right)
$$

where $G_{2}$, the quadratic term of $F$, satisfies the null condition, $G_{3}$ is a cubic term of $F$, and 


$$
H\left(u, u^{\prime}\right)=O\left(|u|^{4}+\left|u^{\prime}\right|^{4}\right) \text { near }\left(u, u^{\prime}\right)=0
$$

(A9) In addition, $G_{3}$ satisfies the null condition.

Then $F$ can be written as

$$
F\left(u, u^{\prime}\right)=c Q(u, u)+P\left(u, u^{\prime}\right) Q(u, u)+H\left(u, u^{\prime}\right)
$$

where $c$ is a constant, $Q(u, u)=\left(\partial_{t} u\right)^{2}-\sum_{i=1}^{2}\left(\partial_{i} u\right)^{2}$ as in section $2, P$ is a linear combination of $u$ and $\left(\partial_{a} u\right)_{a=0,1,2}$, and $H\left(u, u^{\prime}\right)=O\left(|u|^{4}+\left|u^{\prime}\right|^{4}\right)$ satisfying $H(u, 0)=O\left(|u|^{5}\right)$.

Let $u$ satisfies (5.1)-(5.2), and let $v=(1-\exp (-c u)) / c$. (See [2].) Then

$$
\begin{aligned}
\square v & =(1-c v)\{\square u-c Q(u, u)\} \\
& =(1-c v)\left\{P\left(u, u^{\prime}\right) Q(u, u)+H\left(u, u^{\prime}\right)\right\}
\end{aligned}
$$

Define

$$
\begin{aligned}
\tilde{F}\left(v, v^{\prime}\right)= & \tilde{F}\left(v,\left(\partial_{a} v\right)_{a=0,1,2}\right) \\
= & \frac{1}{(1-c v)} P\left(-c^{-1} \log (1-c v),\left((1-c v)^{-1} \partial_{a} v\right)_{a=0,1,2}\right) Q(v, v) \\
& \quad+(1-c v) H\left(-c^{-1} \log (1-c v),\left((1-c v)^{-1} \partial_{a} v\right)_{a=0,1,2}\right)
\end{aligned}
$$

Then $v$ satisfies

$$
\square v=\tilde{F}\left(v, v^{\prime}\right),
$$

with initial data

$$
v(0)=(1-\exp (-\varepsilon c f)) / c, \partial_{t} v(0)=\varepsilon g \exp (-\varepsilon c f) .
$$

One can verify that $\tilde{F}\left(v, v^{\prime}\right)$ satisfies (A1)-(A4). So Theorem 1 and Remark 2 imply that there exists a unique solution to (5.4)-(5.5) for $0 \leq t<+\infty$, if $\varepsilon$ is sufficiently small. Therefore the Cauchy problem (5.1)-(5.2) also has a global solution.

Similarly, if we only assume (A6)-(A8) and not (A9), we can show that the reduced equation (5.4) satisfies (A1)-(A3). So the result in Remark 1 implies that there exists a unique solution for $0 \leq t<\exp \left\{A \varepsilon^{-2}\right\}$ with some constant $A>0$, if $\varepsilon$ is sufficiently small.

Summing up, we have proved the following : 
Theorem 2. Assume (A6)-(A8). Then there exist some constants $\varepsilon_{0}>0$ and $A>0$, such that for any $\varepsilon \leq \varepsilon_{0}$, there exists a unique solution $u(t, x)$ to (5. 1)-(5.2) for $0 \leq t<\exp \left\{A \varepsilon^{-2}\right\}$.

If we assume (A9) in addition, then there exists $\varepsilon_{0}>0$ such that for any $\varepsilon$ $\leq \varepsilon_{0}$, the Cauchy problem (5.1)-(5.2) has a global solution.

\section{Acknowledgements}

The author would like to express his gratitude to Professors Y. Ohya and S. Tarama for their helpful suggestions and constant encouragement.

\section{References}

[1] Christodoulou, D., Global solutions of nonlinear hyperbolic equations for small initial data, Comm. Pure Appl. Math., 39(1986), 267-282.

[2] Godin, P., Lifespan of semilinear wave equations in two space dimensions, Preprint.

[3] Hörmander, L., The Analysis of Linear Partial Differential Operators I, Springer-Verlag, New York, 1983.

[4] $-L^{1}, L^{\infty}$ estimates for the wave operator, in Analyse Mathématique et Applications, Contributions en l'Honneur de J. L. Lions, Gauthier-Villars, Paris, 1988, 211-234.

[5] John, F., Existence for large times of strict solutions of nonlinear wave equations in three space dimensions for small initial data, Comm. Pure Appl. Math., 40(1987), 79-109.

[6] Klainerman, S., Global existence for nonlinear wave equations, Comm. Pure Appl. Math., 33 (1980), 43-101.

[7] Uniform decay estimates and the Lorentz invariance of the classical wave equation, Comm. Pure Appl. Math., 38(1985), 321-332.

[8] The null condition and global existence to nonlinear wave equations, Lectures in Applied Math., 23(1986), 293-326.

[9] Kovalyov, M., Long-time behavior of solutions of a system of nonlinear wave equations, Commun. in Partial Differential Equations, 12(1987), 471-501.

[10] Lindblad, H., On the lifespan of solutions of nonlinear wave equations with small initial data, Comm. Pure Appl. Math., 43(1990), 445-472.

[11] Mizohata, S., The Theory of Partial Differential Equations, Cambridge University Press, London, 1973.

[12] Li Ta-tsien, and Yu Xin, Life-span of classical solutions to fully nonlinear wave equations, Comm. in Partial Differential Equations, 16(1991), 909-940.

[13] Li Ta-tsien, Yu Xin, and Zhou Yi, Problème de Cauchy pour les équations des ondes non linéaires avec petites données initiales, C. R. Acad. Sci. Paris, 312, Série I (1991), 337-340. 
